\title{
Underdiagnosis of cardiopulmonary involvement in patients with idiopathic inflammatory myopathies
}

\author{
Aleksandra Opinc ID, Olga Brzezińska, Joanna Makowska ID \\ Department of Rheumatology, Medical University of Lodz, Poland
}

\begin{abstract}
Objectives: In the course of idiopathic inflammatory myopathies internal organs, including heart and lungs, can be involved. Cardiopulmonary complications significantly alter the course of the disease, leading to poorer prognosis. A lack of clear guidelines on the assessment of internal organ involvement in the course of myositis increases the risk of underdiagnosis. The aim of the study was to evaluate the incidence of clinical symptoms indicative of cardiovascular and pulmonary involvement in patients with myositis, and the impact of these ailments on daily living.

Material and methods: A self-designed online survey was distributed via online support groups and community forums for patients with idiopathic inflammatory myopathies. The questionnaire contained inquiries about demographical data, clinical symptoms, including symptoms indicative of cardiopulmonary involvement, as well as the standardised Health Assessment Questionnaire. Respondents were divided according to concomitant diseases into a subgroup diagnosed with cardiopulmonary diseases and a subgroup without such comorbidities. The prevalence of cardiopulmonary symptoms was compared between the subgroups. The impact of cardiopulmonary symptoms on the degree of disability and daily functioning was assessed.

Results: In total, 370 patients were included in the study group. The most commonly symptoms included dyspnoea during exercise, palpitations and ankle oedema during daily activities. Cardiopulmonary symptoms were frequent in respondents diagnosed with cardiopulmonary diseases and in patients declaring no comorbidities of the heart and lungs. Intensity of chest pain, tolerance of physical activity, and fatigue were comparable in both of the study subgroups. The degree of disability was higher in respondents with concomitant cardiovascular and/or pulmonary comorbidities, but only dry cough and ankle oedema impacted the results.

Conclusions: Clinical symptoms indicative of heart and lung involvement occur frequently in patients with idiopathic inflammatory myopathies; however, cardiopulmonary complications seem to be relatively rarely detected. Active screening for cardiopulmonary involvement is recommended.
\end{abstract}

Key words: idiopathic inflammatory myopathies, heart failure, interstitial lung disease, underdiagnosis.

\section{Introduction}

Idiopathic inflammatory myopathies (IIM) are a large and highly heterogeneous group of rare connective tissue diseases. The mean incidence of IIM in the adult population has been estimated as 17.6 per million per year [1], with a clear increasing trend observed over time [2]. Although skeletal muscle involvement remains the hallmark of myositis, systemic inflammation underlies IIM, leading to multi-organ symptoms $[3,4]$. In the course of IIM, involvement of the skin, joints, respiratory tract, cardiovascular system and gastrointestinal tract is observed [5].

Recent data suggest frequent incidence of cardiovascular involvement in the course of IIM. According to the international registry, it affects approximately $9 \%$ of

\footnotetext{
Address for correspondence:

Joanna Makowska, Department of Rheumatology, Medical University of Lodz, 113 St. Żeromski St., 90-549 Lodz, Poland,

e-mail: joanna.makowska@umed.lodz.pl
}

Submitted: 21.09.2021; Accepted: 11.10.2021 
patients with myositis [6]. Cardiovascular complications seem to be the most frequent in overlapping syndromes (especially in scleromyositis) and the least prevalent in inclusion body myositis [6]. Clinically apparent cardiovascular involvement usually presents as congestive heart failure [7].

Numerous studies indicate that subclinical cardiac involvement occurs far more frequently than symptomatic presentation: depending on the study it was detected in $13-72 \%$ of patients with IIM [7-9]. Most of the asymptomatic abnormalities can be easily detected with the use of non-invasive methods such as electrocardiography or echocardiography. Although non-specific ST-T or T-wave changes seem to be the most prevalent, potentially life-threatening arrhythmias have also been detected in patients with myositis [10].

Pulmonary involvement in the course of IIM usually manifests with interstitial lung disease (ILD) [11]. In some of the patients, interstitial lesions may proceed the onset of the fully symptomatic myositis [11]. Noteworthy, approximately 1 in 5 patients have an asymptomatic course of ILD, which justifies the need for active screening [12].

Involvement of the cardiovascular and pulmonary systems not only alters the clinical presentation but most of all is considered as a highly unfavourable prognostic factor, which contributes to significantly higher mortality $[13,14]$. Pulmonary and cardiac complications, along with neoplastic diseases, remain the leading causes of death in the course of IIM [10].

The risk of developing coronary artery disease and myocardial infarction in patients with polymyositis/dermatomyositis have been reported to be at least 3-fold higher than in the general population [15-17]. Furthermore, people with IIM may be at greater risk of developing thromboembolism and pulmonary hypertension $[10,18]$. Early diagnosis of cardiopulmonary lesions allows the implementation of an appropriately adjusted treatment.

The aim of this study was to evaluate the incidence of clinical symptoms indicative of the cardiovascular and pulmonary involvement in patients with IIM, as well as to assess the impact of these ailments on daily living and the degree of disability.

\section{Material and methods}

The study is a cross-sectional descriptive survey of patients with IIM. The questionnaire used had a digital form and was designed by the authors of the study. The questionnaire was distributed via international online support groups and community forums to English-speaking patients with IIM.
To ensure the human subject rights, the survey was anonymous and no data enabling patient identification were collected. Filling in the questionnaire was voluntary. Before answering the questions, information concerning the purpose of the study and the range of questions was displayed. Due to the anonymous nature of the study and the remote form of data collection, separate consents from the participants were not required and completion of the questionnaire was tantamount to consent to participate in the study. Respondents could resign from completing the survey at any time.

The survey consisted of 40 single-choice and multiple-choice questions with open-ended questions. It included inquiries about demographical data (age, gender, disease duration), concomitant diseases, frequency of clinical symptoms including predominantly symptoms indicative of cardiovascular and pulmonary involvement (single choice questions with options of gradable frequency - "no", "yes, a few times a month", "yes, a few times a week", "yes, every day"), intensity of gradable symptoms.

Symptoms were considered as present if they occurred with a frequency of at least a few times a month. The mean intensities of the chest pain, fatigue, and tolerance of physical activity were assessed with VAS 1-10, where 1 indicated the lowest and 10 the highest intensity.

At the end of the survey the respondents were asked to fill in the standardized Health Assessment Questionnaire - disability index (HAQ-DI), which consists of 20 questions concerning the activities of daily living, divided into 8 categories (dressing and grooming, arising, eating, walking, hygiene, reaching, gripping, and daily activities).

In each of the categories the patients were asked about their ability to perform several daily duties, which were scored as follows: 0 points for performing the tasks "without any difficulty", 1 point for "with some difficulty", 2 points for the answer "with much difficulty", and 3 points for "unable to do". The final score for each of the categories was equal to the highest score obtained in this category. Respondents were also asked to specify whether they require the aid of the caregivers or usage of the facilitating devices while performing different daily duties. Based on the answers in HAQ-DI, standard and alternative disability indexes (SDI and ADIs) were counted following the instructions for the Health Assessment Questionnaire by International Myositis Assessment and Clinical Studies [19, 20].

Alternative disability index was counted by adding final scores for all of the 8 categories and dividing the sum by 8 . In order to count SDI, the final scores were adjusted - if the final score for the category was 0 or 1 , 


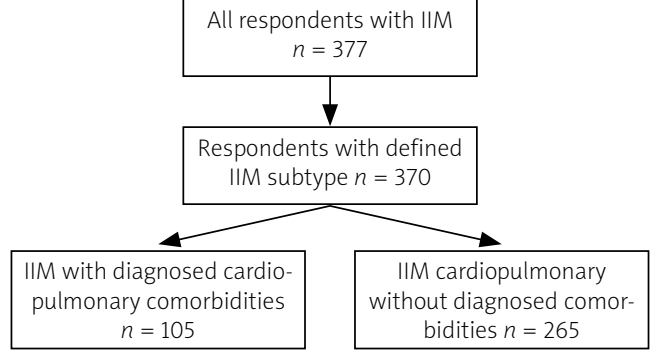

IIM - idiopathic inflammatory myopathies, $n$-number of people.

Fig. 1. Process of inclusion to the study groups.

but the patient declared needing aid of the caregivers or using facilitating devices, the score for that category was changed to 2 points. Final scores of 2 or 3 were not altered. Standard disability index was obtained by adding the adjusted final scores and dividing the sum by the number of categories. Standard disability index and ADI of 0 reflect "no disability", scores of 0 to 1 represent "mild to moderate difficulty", 1 to 2 indicate "moderate to severe disability", while scores between 2 and 3 reflect "severe to very severe disability".

Inclusion criteria to the study group included voluntary filling of the questionnaire, self-reported diagnosis of IIM subtype, and providing answers to each of the obligatory questions. Exclusion criteria included lack of the awareness of the exact diagnosis or incomplete filling of the questionnaire. Due to the nature of the study (anonymous questionnaire), estimation of the sample size was not performed. The size of the study group depended on the number of people who filled in the questionnaire, which could not be estimated at the planning stage.

Participants included in the study group were divided into 2 groups: a subgroup of patients with IIM and cardiopulmonary comorbidities (such as heart failure, coronary artery disease/ischemic heart disease, arrhythmia, atrioventricular blocks, implanted pacemaker, asthma, chronic obstructive pulmonary disease, interstitial lung disease, pulmonary hypertension) and a subgroup of patients with myositis but not diagnosed with such comorbidities (Fig. 1).

Isolated hypertension, as a clinically asymptomatic disorder, was not included in the cardiopulmonary comorbidities. The occurrence and intensity of symptoms indicative of cardiopulmonary involvement was compared between the 2 subgroups. The degree of disability, reflected by SDI and ADI, was compared between the respondents with and without different cardiopulmonary symptoms in order to assess which of the manifestations have the strongest impact on daily living.

The acquired data were analysed statistically with the use of STATISTICA 13.1 software. Statistical meth- ods were selected appropriately according to the type of data: the normality of the data was assessed with the Shapiro-Wilk test, the equivalence of the group was tested by $\chi^{2}$ test, and for ordinal variables the Mann-Whitney $U$ test was used.

\section{Results}

The questionnaire was completed by 377 respondents, 370 of whom were included in the study group. Patients unaware of the subtype of IIM diagnosed in them $(1.86 \%, n=7)$ were excluded to eliminate the risk of completing the questionnaire by individuals suffering from myopathies other than IIM (Fig. 1).

The characteristics of the study participants are presented in Table I. Of note, the subgroups of participants diagnosed and non-diagnosed with cardiopulmonary comorbidities were found to be remarkably similar in terms of age and gender, disease subtypes, and disease duration (Table I). Respondents with defined cardiopulmonary diseases were more often affected by reflux disease $(p<0.0001)$ and hyperthyroidism $(p=0.036)$.

The most commonly reported symptoms included dyspnoea at exercise (71.08\% of the cohort), dyspnoea during daily activities (51.35\%), palpitations (51.89\%), and ankle oedema (51.62\%). Dry cough occurred in $48.92 \%$, while a feeling of too rapid or irregular heart rate were noted by, respectively, $45.14 \%$ and $42.16 \%$ of the patients with myositis.

Approximately 1 in 3 respondents declared chest pain or shortness of breath at rest. Of note, symptoms indicative of cardiopulmonary involvement were frequent not only in patients diagnosed with concomitant disorders of the heart and lungs, but also in respondents without stated diagnosis of such comorbidities (Fig. 2).

Symptoms such as dyspnoea during daily activities, orthopnoea, chest pain, palpitations, feeling of too rapid heart rate, pauses, and nycturia occurred with a similar frequency in both subgroups of patients with IIM ( $p>0.05$, Fig. 2).

The mean intensity of chest pain, assessed with VAS 1-10 scale, reached 3.21 in the subgroup of respondents with defined concomitant cardiopulmonary diseases and 2.94 in patients not diagnosed with such comorbidities ( $p=0.303$ ). People with myositis presented decreased tolerance to physical activity, but the difference between subgroups of respondents with and without cardiopulmonary diseases did not reach statistical significance (respectively, 7.13 in the subgroup diagnosed with cardiopulmonary comorbidities and 6.74 in the subgroup with no diagnosis of heart and lung diseases, $p=0.124)$. Similarly, the intensity of fatigue was comparable in the subgroups with and without cardiopulmonary disorders (respectively, 6.48 vs. 6.31, $p=0.387$ ). 
Table I. Characteristics of the respondents included to the study group

\begin{tabular}{|c|c|c|c|}
\hline Respondents' characteristics & $\begin{array}{c}\text { Respondents with IIM } \\
\text { diagnosed with CP diseases } \\
{[\%](N=105)}\end{array}$ & $\begin{array}{c}\text { Respondents with IIM not } \\
\text { diagnosed with CP diseases } \\
{[\%](N=265)}\end{array}$ & $p$-values \\
\hline \multicolumn{4}{|c|}{ Gender } \\
\hline Female & $64.76(n=68)$ & $68.68(n=182)$ & ns \\
\hline Male & $35.24(n=37)$ & $31.32(n=83)$ & ns \\
\hline \multicolumn{4}{|c|}{ Age structure } \\
\hline$<18$ years old & $0.95(n=1)$ & $1.89(n=5)$ & ns \\
\hline $18-25$ years old & $1.90(n=2)$ & $4.15(n=11)$ & ns \\
\hline 26-40 years old & $8.57(n=9)$ & $14.72(n=39)$ & ns \\
\hline $41-65$ years old & $47.62(n=50)$ & $47.92(n=127)$ & ns \\
\hline $66-75$ years old & $31.43(n=33)$ & $21.13(n=56)$ & ns \\
\hline 76 years old and more & $9.52(n=10)$ & $10.19(n=27)$ & ns \\
\hline \multicolumn{4}{|c|}{ Subtype of IIM } \\
\hline Dermatomyositis & $28.57(n=30)$ & $35.09(n=93)$ & ns \\
\hline Polymyositis & $20.00(n=21)$ & $14.72(n=39)$ & ns \\
\hline Inclusion body myositis & $40.95(n=43)$ & $36.60(n=97)$ & ns \\
\hline Overlapping syndromes & $8.57(n=9)$ & $10.94(n=29)$ & ns \\
\hline Juvenile myositis & $1.90(n=2)$ & $2.64(n=7)$ & ns \\
\hline \multicolumn{4}{|c|}{ Disease duration } \\
\hline Less than 3 months & $3.81(n=4)$ & $2.64(n=7)$ & ns \\
\hline $3-6$ months & $1.90(n=2)$ & $4.53(n=12)$ & ns \\
\hline $6-12$ months & $5.71(n=6)$ & $6.04(n=16)$ & ns \\
\hline $1-3$ years & $18.10(n=19)$ & $23.02(n=61)$ & ns \\
\hline $3-5$ years & $21.90(n=23)$ & $16.98(n=45)$ & ns \\
\hline More than 5 years & $48.57(n=51)$ & $46.79(n=124)$ & ns \\
\hline \multicolumn{4}{|c|}{ Comorbidities (listed from the most prevalent) } \\
\hline Smokers or former smokers & $45.71(n=48)$ & $40.75(n=108)$ & ns \\
\hline Hypertension & $37.14(n=39)$ & $29.43(n=78)$ & ns \\
\hline Reflux disease & $40.00(n=42)$ & $20.00(n=53)$ & $<0.0001$ \\
\hline Hypothyroidism & $20.00(n=21)$ & $13.58(n=36)$ & ns \\
\hline Diabetes mellitus type 2 & $15.24(n=16)$ & $10.19(n=27)$ & ns \\
\hline Asthma* & $33.33(n=35)$ & - & - \\
\hline Arrhythmia* & $32.38(n=34)$ & - & - \\
\hline Neoplasm & $11.43(n=12)$ & $6.42(n=17)$ & ns \\
\hline Coronary artery disease/ischaemic heart disease* & $19.05(n=20)$ & - & - \\
\hline Chronic obstructive pulmonary disease* & $15.24(n=16)$ & - & - \\
\hline Heart failure* & $10.48(n=11)$ & - & - \\
\hline Venous thrombosis & $2.86(n=3)$ & $3.02(n=8)$ & ns \\
\hline Interstitial lung disease ${ }^{\star}$ & $8.57(n=9)$ & - & - \\
\hline Hyperthyroidism & $3.81(n=4)$ & $0.85(n=2)$ & 0.036 \\
\hline Peptic ulcers & $2.86(n=3)$ & $0.75(n=2)$ & ns \\
\hline Pulmonary hypertension* & $4.76(n=5)$ & - & - \\
\hline Atrioventricular block* & $3.81(n=4)$ & - & - \\
\hline Diabetes mellitus type 1 & $1.90(n=2)$ & $0.75(n=2)$ & ns \\
\hline Implanted pacemaker* & $3.81(n=4)$ & - & - \\
\hline Depression & $1.90(n=2)$ & $0.75(n=2)$ & ns \\
\hline Migraines & $0.95(n=1)$ & $1.12(n=3)$ & ns \\
\hline None & - & $29.06(n=77)$ & - \\
\hline
\end{tabular}

CP-cardiopulmonary, IIM - idiopathic inflammatory myopathies; $n$ - number of people, ${ }^{*}$ comorbidity included as a concomitant cardiopulmonary disease. 

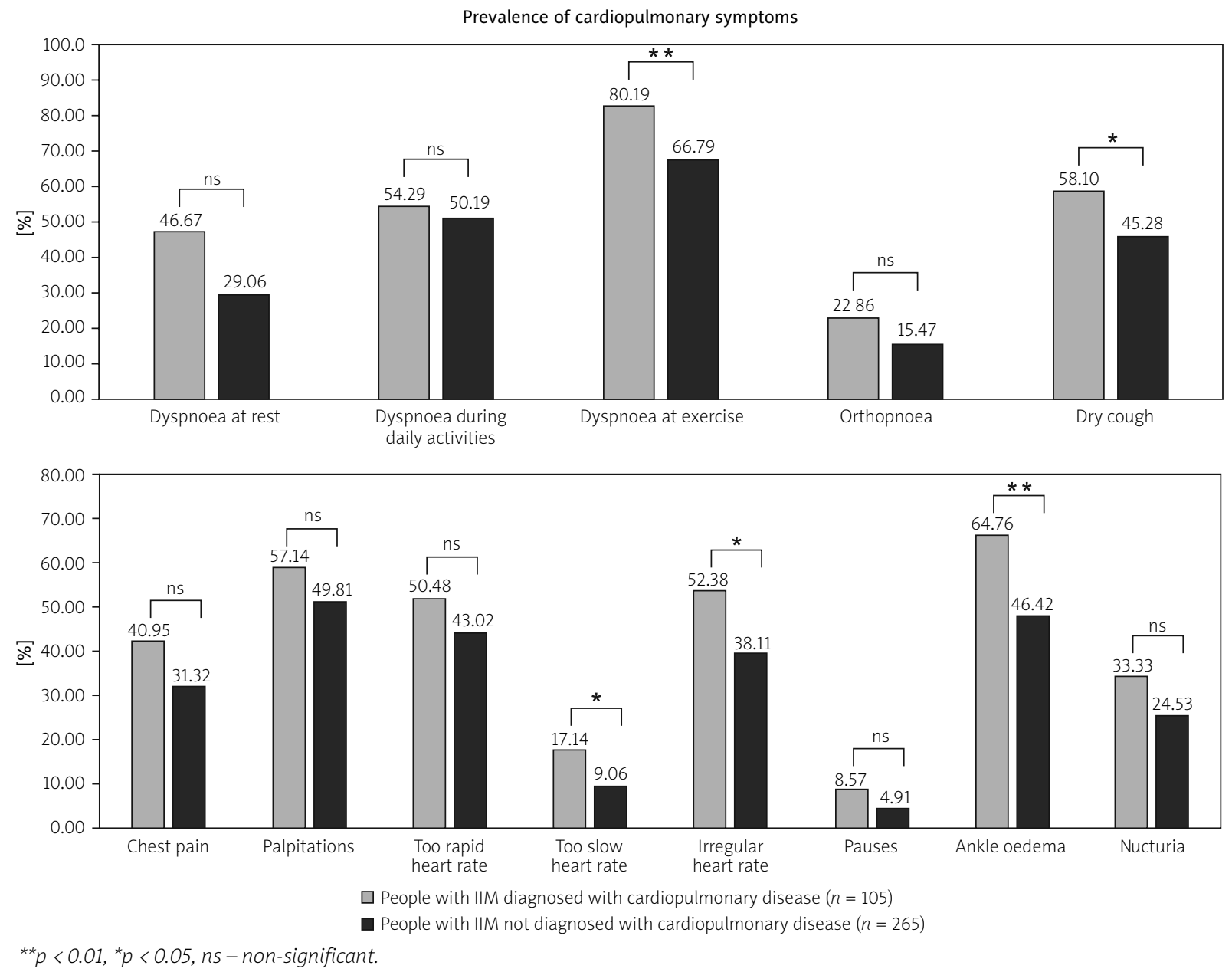

Fig. 2. Comparison of the incidence of symptoms in the subgroup of patients with and without concomitant cardiopulmonary diseases.

Regardless of the comorbidities, symptoms such as dyspnoea, chest pain, palpitations, and too rapid heart rate were statistically more often observed in patients with dermatomyositis, polymyositis, and overlapping syndromes as compared to respondents with inclusion body myositis. Conversely, no statistically significant differences were noted regarding the frequency of bradycardia, pauses, dry cough, ankle oedema, and nycturia in patients with different subtypes of IIM.
The degree of disability, reflected by SDI and ADIs, was higher in respondents with concomitant cardiovascular and/or pulmonary comorbidities as compared to patients with IIM not diagnosed with heart and lung diseases (SDI 1.66 vs. 1.35, $p=0.004$ and ADI 1.51 vs. 1.23, $p=0.002)$.

Patients with dry cough and ankle oedema presented higher values of SDI and ADI as compared to respondents without those symptoms (Table II). No statistically

Table II. Cardiopulmonary symptoms impacting daily functioning

\begin{tabular}{|lcccc|}
\hline Symptom & & Respondents with symptom & Respondents without symptom & $p$-value \\
\hline Ankle oedema & Mean SDI & $1.71 \pm 0.81$ & $1.15 \pm 0.86$ & $<0.0001$ \\
\cline { 2 - 5 } & Mean ADI & $1.58 \pm 0.84$ & $1.03 \pm 0.83$ & $<0.0001$ \\
\hline \multirow{2}{*}{ Dry cough } & Mean SDI & $1.55 \pm 0.85$ & $1.33 \pm 0.89$ & 0.013 \\
\cline { 2 - 5 } & Mean ADI & $1.41 \pm 0.85$ & $1.22 \pm 0.89$ & 0.023 \\
\hline
\end{tabular}

$A D I$ - alternative disability index, SDI - standard disability index. 
significant differences in the degree of disability were observed for the remaining cardiopulmonary symptoms.

\section{Discussion}

The presented results display a high prevalence of symptoms indicative of cardiovascular and/or pulmonary involvement in patients with IIM. Many of the symptoms possibly indicating cardiovascular involvement are non-specific and could be related also to pulmonary disorders; therefore, in most of the patients, distinguishing between cardiac or pulmonary complications is difficult and requires additional tests.

One such symptom is dyspnoea, which could originate from both cardiac and pulmonary conditions. We noted a high prevalence of shortness of breath, especially occurring during physical exercise. It remains in line with a study by Diederichsen et al. [21], in which $57 \%$ of 76 patients with IIM complained of dyspnoea.

Considering prospective cohorts, dyspnoea seems to be less prevalent because it occurred only in $10.76 \%$ of 195 people with myositis [22]. Orthopnoea, typically associated with left ventricular heart failure, occurred in $17.57 \%$ of the respondents from our study. Similarly, in a small prospective study, $12 \%$ out of 26 patients with polymyositis suffered from orthopnoea [23].

Cases of peripheral oedema have been previously reported in the literature [22], but not with such a high frequency as in our study. Comparing to the previous observations, we noted a higher incidence of palpitations, which could indicate arrhythmia. In a study by Diederichsen et al. [21] 34\% of adults with dermatomyositis or polymyositis complained of palpitations, and a comparable prevalence of chest pounding was observed by Taylor et al. [23].

Conversely, the analysis of prospective cohorts revealed a relatively low incidence of palpitations [22]. Similarly, previously reported data suggested a lower incidence of chest pain than noted in our results $[22,23]$. Interestingly, the frequency of non-specific cardiopulmonary manifestations seems to be more prevalent in retrospective cohorts as compared to the prospective ones [22]. The discrepancy of the data in the prospective and retrospective observations suggests a large time delay to the onset of cardiac/pulmonary symptoms, resulting in missing the records in prospective short-term observations.

Of note, manifestations from the cardiovascular system and/or respiratory tract were common not only in patients with IIM and stated diagnosis of cardiopulmonary comorbidities, but also in the subgroup not diagnosed with disorders of the heart and lungs, strongly suggesting underdiagnosis of concomitant comorbidities in the second group. Despite the high incidence of symp- toms from the heart and lungs, in less than $30 \%$ of the patients from our study a diagnosis of cardiac or pulmonary disorder was stated, which is remarkably lower than the incidence observed in international registries [6].

Indeed, in recent years attention has been attracted to cardiovascular involvement as a possible consequence of myositis. Symptomatic involvement of the heart is considered to be less prevalent than subclinical involvement, which has been well documented in numerous studies based on electrocardiography and echocardiography [10]. The most common clinical presentation of cardiac involvement in the course of IIM is congestive heart failure, the prevalence of which differs significantly depending on the study but reaches as much as 32-77\% [7].

In our study, a diagnosis of heart failure was stated in only $2.97 \%$ of the respondents, while in $5.41 \%$ coronary artery disease/ischaemic heart disease was recognized. Considering the frequency of declared symptoms indicative of cardiac involvement, it seems highly probable that some of the respondents were not diagnosed accurately.

Similarly, frequent involvement of the respiratory tract has been well documented in the course of IIM $[6,11]$. The most common presentation is interstitial lung disease, which is observed in approximately $30 \%$ of people with myositis; pulmonary hypertension or pleural lesions may also occur $[6,11]$.

The prevalence of interstitial lesions may differ depending on the subtype of IIM. It is especially common in the course of antisynthetase syndrome, being the predominant presentation in some patients with this subtype of IIM [24].

Of note, in our study only $2.43 \%$ of the respondents declared being diagnosed with interstitial lung disease, which seems to be significantly underestimated, especially in light of the very high incidence of symptoms such as dry cough or dyspnoea. These observations are alarming because they may suggest that in a significant proportion of patients with IIM, the evaluation of cardiovascular and pulmonary involvement is not even performed.

The average tolerance of physical activity was reported to be significantly decreased in patients with myositis, regardless of the diagnosis of concomitant cardiopulmonary diseases. Similarly, the degree of fatigue was not associated with such comorbidities. Although most cardiopulmonary symptoms did not contribute to a greater degree of disability, ankle oedema and dry cough seem to exert a negative impact on daily functioning.

This led to the conclusion that some factors other than the involvement of the cardiac system and lungs 
contribute to a greater extent to the deterioration of the quality of life. Due to the multitude of collected data, a detailed analysis of the factors aggravating disability, based on the HAQ-DI part of the questionnaire, was presented in a separate publication [25].

Cardiac and pulmonary involvement in the course of IIM are considered as highly unfavourable prognostic factors and remain the leading causes of death [14]. Patients with cardiac lesions in the course of polymyositis/ dermatomyositis were characterised by poorer 8-year survival as compared to patients without cardiovascular lesions [13].

Increased risk of myocardial infarction in the course of IIM has been documented [16, 26]. Such risk lasts for at least several years, but it is the highest during the first year after the diagnosis of myositis [17]. Interestingly, this tendency cannot be fully explained by inflammatory-induced accelerated formation of atherosclerotic plaques because the prevalence of atherosclerosis in patients with IIM was found to be comparable to that in the general population [26]. Most deaths caused by cardiovascular involvement were reported after 5 years of disease duration, while pulmonary complications occurred mostly during the first year after the diagnosis of IIM [14].

Our results entail certain implications for clinical practice. In the shield of described data, it seems justified to actively screen for cardiopulmonary complications in patients with IIM, including those not presenting symptoms from the heart or lungs. Despite increasing awareness of frequent organ involvement, clear guidelines for the diagnosis and monitoring of the complications in the course of IIM have not been developed yet. Therefore, some clinicians may remain unaware of the need to actively screen for internal organ involvement in this group of patients.

According to the literature, high-resolution computed tomography of the chest and pulmonary function tests are highly recommended in patients with IIM, to assess the efficiency of the respiratory system [27]. Due to the frequent subclinical myocardial involvement, routine ECG evaluation and echocardiography are justified, even in individuals without cardiac symptoms [27]. Our results suggest that involvement of the cardiovascular and pulmonary systems are independent of age and disease duration. Therefore, it seems advisable to start the screening at the early stage of the disease and not limit it to people with clinically severe myositis. Algorithms on the frequency of cardiopulmonary evaluation in the course of IIM are highly anticipated.

Early detection of cardiopulmonary complications may be beneficial because it enables the treatment of the underlying autoimmune disease to be adjusted, and it leads to implementation of the organ-specific therapy aimed at improving the functioning of the heart and lungs, thus enhancing the chances of a favourable outcome.

According to the literature, myositis-associated ILD is usually treated with a combination of glucocorticoids and immunomodulating drugs, among which cyclophosphamide, calcineurin inhibitors, and rituximab may be especially useful $[28,29]$. Several studies support the use of mycophenolate mofetil and azathioprine [29]. Some patients with ILD in the course of IIM require oxygen therapy or even lung transplantation due to severe, irreversible lesions [24]. Data on the effectiveness of various therapeutic options in patients with cardiac complications in the course of IIM remain less consistent. Implementation of glucocorticosteroids with immunosuppressive drugs was reported to exert a beneficial effect [30].

Positive effects have been documented for rituximab and azathioprine [16, 31]. In some patients with myositis, due to severe rhythm disturbances, pacemaker implantation or cardioversion were needed as well as immunosuppressive drugs [32-34].

However, most of the data is based on retrospective observations and small-group studies. Randomized controlled trials are needed to assess the effectiveness of individual therapeutic regimens.

\section{Study limitations}

The study has some certain limitations. The part of the questionnaire written by the authors was not validated. Self-reporting of the diagnosis remains the main limitation of the study. The questionnaire was performed remotely by means of an online form without a direct examination by a physician.

Although the questionnaire was addressed to English-speaking patients, we cannot exclude the possibility that some of the respondents might have had a lower command of English, which could have influenced the understanding of the stated questions.

Because we do not know how many people viewed the survey, the response rate remains unknown. A lack of sample size estimation limits the credibility of the statistical analysis. However, strategies have been taken to enable the highest possible level of reliability. The questionnaire was sent only to the groups of patients with IIM and no other autoimmune diseases.

Furthermore, the survey included a single-choice question concerning the subtype of IIM, and respondents who did not know their exact diagnosis were excluded from the study group to eliminate the possibility of filling in the questionnaire by people suffering 
from other types of myopathies. The Health Assessment Questionnaire - disability index was chosen to assess daily functioning of the respondents because it is a standardized tool with a well-documented use in IIM, and it is recommended by scientific societies as part of the "Core Set Measures" aimed at assessing the course of the disease, response to treatment, and quality of life in patients with IIM $[35,36]$.

\section{Conclusions}

Clinical symptoms indicative of heart and lung involvement occur frequently in patients with IIM; however, cardiopulmonary complications seem to be relatively rarely detected. Regular evaluation of internal organ involvement is essential and advisable in every patient suffering from IIM. Underdiagnosis of cardiopulmonary complications may lead to severe consequences because they remain the leading causes of death.

Long-term prospective observations on large groups could shed a novel light on cardiopulmonary complications and their impact on daily functioning and mortality. Close cooperation of rheumatologists, pulmonologists, and cardiologists is necessary to provide comprehensive care for patients with IIM.

The authors declare no conflict of interest.

\section{References}

1. Lundberg IE, Tjärnlund A, Bottai M, et al. 2017 European League Against Rheumatism/American College of Rheumatology classification criteria for adult and juvenile idiopathic inflammatory myopathies and their major subgroups. Arthritis Rheumatol 2017; 69: 2271-2282, DOI: 10.1002/art.40320.

2. Meyer A, Meyer N, Schaeffer M, et al. Incidence and prevalence of inflammatory myopathies: a systematic review. Rheumatology (Oxford) 2015; 54: 50-63, DOI: 10.1093/rheumatology/keu289.

3. Vencovský J, Alexanderson H, Lundberg IE. Idiopathic inflammatory myopathies. Rheum Dis Clin North Am 2019; 45: 569581, DOI: 10.1016/j.rdc.2019.07.006.

4. Lundberg IE, de Visser M, Werth VP. Classification of myositis. Nat Rev Rheumatol 2018; 14: 269-278, DOI: 10.1038/ nrrheum.2018.41.

5. Cavazzana I, Fredi M, Selmi C, et al. The Clinical and histological spectrum of idiopathic inflammatory myopathies. Clin Rev Allergy Immunol 2017; 52: 88-98, DOI: 10.1007/s12016015-8517-4.

6. Lilleker JB, Vencovsky J, Wang G, et al. The EuroMyositis registry: an international collaborative tool to facilitate myositis research. Ann Rheum Dis 2018; 77: 30-39, DOI: 10.1136/annrheumdis-2017-211868.

7. Zhang L, Wang GC, Ma L, Zu N. Cardiac involvement in adult polymyositis or dermatomyositis: a systematic review. Clin Cardiol 2012; 35: 686-691, DOI: 10.1002/clc.22026.
8. Bienias P, Ciurzyński M, Korczak D PP. Zaburzenia rytmu i przewodzenia u osób z chorobami układowymi tkanki łącznej. Kardiol Pol 2008; 66: 194-199.

9. Lundberg IE. The heart in dermatomyositis and polymyositis. Rheumatology (Oxford) 2006; 45(Suppl 4): iv18-21, DOI: 10.1093/rheumatology/kel311.

10. Opinc AH, Makowski MA, Łukasik ZM, Makowska JS. Cardiovascular complications in patients with idiopathic inflammatory myopathies: does heart matter in idiopathic inflammatory myopathies? Heart Fail Rev 2021; 26: 111-125, DOI: 10.1007/s10741-019-09909-8.

11. Lega JC, Reynaud Q, Belot A, et al. Idiopathic inflammatory myopathies and the lung. Eur Respir Rev 2015; 24: 216-238, DOI: 10.1183/16000617.00002015.

12. Cavagna L, Trallero-Araguás E, Meloni F, et al. Influence of antisynthetase antibodies specificities on antisynthetase syndrome clinical spectrum time course. J Clin Med 2019; 8: 2013, DOI: 10.3390/jcm8112013.

13. Marie I. Morbidity and mortality in adult polymyositis and dermatomyositis. Curr Rheumatol Rep 2012; 14: 275-285, DOI: 10.1007/s11926-012-0249-3.

14. Dankó K, Ponyi A, Constantin T, et al. Long-term survival of patients with idiopathic inflammatory myopathies according to clinical features: a longitudinal study of 162 Cases. Medicine (Baltimore) 2004; 83: 35-42, DOI: 10.1097/01. md.0000109755.65914.5e.

15. Zöller B, Li X, Sundquist J, Sundquist K. Risk of subsequent coronary heart disease in patients hospitalized for immune-mediated diseases: a nationwide follow-up study from Sweden. PLoS One 2012; 7: e33442, DOI: 10.1371/journal. pone.0033442.

16. Tisseverasinghe A, Bernatsky S, Pineau CA. Arterial events in persons with dermatomyositis and polymyositis. J Rheumatol 2009; 36: 1943-1946, DOI: 10.3899/jrheum.090061.

17. Rai SK, Choi HK, Sayre EC, Aviña-Zubieta JA. Risk of myocardial infarction and ischaemic stroke in adults with polymyositis and dermatomyositis: a general population - based study. Rheumatology (Oxford) 2016: 55: 461-469, DOI: 10.1093/ rheumatology/kev336.

18. Carruthers EC, Choi HK, Sayre EC, Aviña-Zubieta JA. Risk of deep venous thrombosis and pulmonary embolism in individuals with polymyositis and dermatomyositis: a general population-based study. Ann Rheum Dis 2016; 75: 110-116, DOI: 10.1136/annrheumdis-2014-205800.

19. Fries JF, Spitz P, Kraines RG, Holman HR. Measurement of patient outcome in arthritis. Arthritis Rheum 1980; 23: 137-145, DOI: 10.1002/art.1780230202.

20. International Myositis Assessment \& Clinical Studies Group. Disease Activity Core Set Measures - Functional Assessment Tools - HAQ, CHAQ, CMAS. Available from: https://www.niehs. nih.gov/research/resources/imacs/diseaseactivity/index.cfm [Accessed: 15.01.2021].

21. Diederichsen LP, Simonsen JA, Diederichsen AC, et al. Cardiac abnormalities in adult patients with polymyositis or dermatomyositis as assessed by noninvasive modalities. Arthritis Care Res (Hoboken) 2016; 68: 1012-1020, DOI: 10.1002/acr.22772.

22. Gupta R, Wayangankar SA, Targoff IN, Hennebry TA. Clinical cardiac involvement in idiopathic inflammatory myopathies: 
a systematic review. Int J Cardiol 2011; 148: 261-270, DOI 10.1016/j.ijcard.2010.08.013.

23. Taylor AJ, Wortham DC, Robert Burge J, Rogan KM. The heart in polymyositis: a prospective evaluation of 26 patients. Clin Cardiol 1993; 16: 802-808, DOI: 10.1002/clc.4960161110.

24. Opinc AH, Makowska JS. Antisynthetase syndrome - much more than just a myopathy. Semin Arthritis Rheum 2021; 51: 72-83, DOI: 10.1016/j.semarthrit.2020.09.020.

25. Opinc AH, Brzezińska OE, Makowska JS. Disability in idiopathic inflammatory myopathies: questionnaire-based study. Rheumatol Int 2019; 39: 1213-1220, DOI: 10.1007/s00296-019-04302-y.

26. Linos E, Fiorentino D, Lingala B, et al. Atherosclerotic cardiovascular disease and dermatomyositis: an analysis of the $\mathrm{Na}$ tionwide Inpatient Sample survey. Arthritis Res Ther 2013; 15 R7, DOI: 10.1186/ar4135.

27. Lundberg IE, Miller FW, Tjärnlund A, Bottai M. Diagnosis and classification of idiopathic inflammatory myopathies. J Intern Med 2016; 280: 39-51, DOI: 10.1111/joim.12524.

28. Mimori T, Nakashima R, Hosono Y. Interstitial lung disease in myositis: clinical subsets, biomarkers, and treatment. Curr Rheumatol Rep 2012; 14: 264-274, DOI: 10.1007/s11926012-0246-6.

29. Long K, Danoff SK. Interstitial lung disease in polymyositis and dermatomyositis. Clin Chest Med 2019; 40: 561-572, DOI: 10.1016/j.ccm.2019.05.004

30. Allanore Y, Vignaux O, Arnaud L, et al. Effects of corticosteroids and immunosuppressors on idiopathic inflammatory myopathy related myocarditis evaluated by magnetic resonance imaging. Ann Rheum Dis 2006; 65: 249-252, DOI: 10.1136/ard.2005.038679.

31. Touma Z, Arayssi T, Kibbi L, Masri AF. Successful treatment of cardiac involvement in dermatomyositis with rituximab. Joint Bone Spine 2008; 75: 334-337, DOI: 10.1016/j.jbspin.2007.05.011.

32. Opinc A, Makowski M, Makowska J. Patients with idiopathic inflammatory myopathies and primary involvement of heart muscle and cardiovascular complications. Biomed J Sci Tech Res 2018; 5: 4467-4470, DOI: 10.26717/bjstr.2018.05.001184.

33. Martti O, Tapio R. Cardiac Involvement in polymyositis. Scand J Rheumatol 1978; 7: 203-208.

34. Lightfoot PR, Bharati S, Lev M. Chronic dermatomyositis with intermittent trifascicular block. An electrophysiologic conduction system correlation. Chest 1977; 71: 413-416, DOI: 10.1378/ chest.71.3.413.

35. Rider LG, Aggarwal R, Machado PM, et al. Update on outcome assessment in myositis. Nat Rev Rheumatol 2018; 14: 303-318, DOI: 10.1038/nrrheum.2018.33.

36. Aggarwal R, Rider LG, Ruperto N, et al. 2016 American College of Rheumatology/European League against rheumatism criteria for minimal, moderate, and major clinical response in adult dermatomyositis and polymyositis: an International Myositis Assessment and Clinical Studies Group/Paediatric Rheumatology International Trials Organisation Collaborative Initiative. Ann Rheum Dis 2017; 76: 792-801, DOI: 10.1136/annrheumdis-2017-211400. 